\title{
PROTÓTIPO PARA A VERIFICAÇÃO DE APRENDIZAGEM EM LABORATÓRIO CLÍNICO DURANTE ESTÁGIO SUPERVISIONADO
}

\author{
PROTOTYPE FOR VERIFICATION OF LEARNING IN CLINICAL LABORATORY \\ DURING SUPERVISED STAGE
}

\section{PROTOTIPO PARA VERIFICACIÓN DE APRENDIZAJE EN LABORATORIO CLÍNICO DURANTE LA ETAPA SUPERVISADA}

\begin{abstract}
Adriano Cavalcante Melo ${ }^{1}$, Ana Luiza Exel ${ }^{2}$, Anne Rose Marques ${ }^{3}$, Newton de Barros Melo Neto $^{4}$, Diego Demerval Medeiros da Cunha Matos ${ }^{5}$, Ranilson Oscar Araújo Paiva ${ }^{6}$
\end{abstract}

\section{RESUMO}

Objetivo: desenvolver protótipo de aplicativo móvel para a verificação de aprendizagem em laboratório clínico durante estágio supervisionado. Método: estudo descritivo acerca do desenvolvimento de aplicativo móvel para auxiliar estudantes e preceptores no processo de aprendizagem baseado na aplicação de questionário likert. A amostra foi selecionada por conveniência e os voluntários foram convidados por meio do WhatsApp a participar da pesquisa, recebendo o link do protótipo e o link do questionário. Após a verificação do protótipo e antes de iniciar as respostas ao questionário, era necessário o aceite do Termo de Consentimento. Após o aceite, os participantes eram direcionados para as respostas ao questionário. Resultados: grupo multiprofissional em saúde, com aproximadamente cinco anos de atuação na preceptoria, com experiência em informática, aprovou, em quase $80 \%$, o uso do sistema em vários aspectos. Conclusões: a utilização de recursos digitais dinâmicos e interativos, considerando a necessidade de um instrumento norteador para os estágios supervisionados em laboratório clínico, mostra-se como alternativa para incrementar o processo de aprendizagem.

Palavras-chave: Aplicativo; Mobile Learning; Tecnologias da Informação.

\section{ABSTRACT}

Objective: develop mobile app prototype for clinical laboratory learning verification during supervised internship. Method: descriptive study about the development of mobile application to assist students and preceptors in the learning process based on the likert questionnaire application. The sample was selected for convenience and volunteers were invited through WhatsApp to participate in the survey, receiving the prototype link and the questionnaire link. After verifying the prototype and before starting the answers to the questionnaire, the Consent Form had to be accepted. After acceptance, participants were

1.2,3 Mestrandos. Universidade Federal de Alagoas. Maceió (AL), Brasil.

4,5,6 Doutores. Universidade Federal de Alagoas. Maceió ( $A L)$, Brasil. 
directed to the answers to the questionnaire. Results: multiprofessional health group, with approximately five years of experience in the preceptorship, with experience in informatics, approved, in almost $80 \%$, the use of the system in several aspects. Conclusion: the use of dynamic and interactive digital resources, considering the need for a guiding instrument for supervised internships in a clinical laboratory, is an alternative to improve the learning process.

Keywords: Application; Mobile Learning; Information Technologies.

\section{RESUMEN}

Objetivos: desarrollar prototipo de aplicación móvil para la verificación de aprendizaje en laboratorio clínico durante la etapa supervisada. Método: estudio descriptivo sobre el desarrollo de aplicaciones móviles para ayudar a los estudiantes y preceptores en el proceso de aprendizaje, basado en la aplicación de cuestionario Likert. La muestra seleccionada fue por conveniencia y los voluntarios fueron invitados a través de WhatsApp a participar en la investigación, recibiendo el link del prototipo y el link del cuestionario. Después de la verificación del prototipo y antes de iniciar las respuestas al cuestionario, era necesario la aceptación del Término de Consentimiento. Después de la aceptación los participantes se dirigían a las respuestas al cuestionario. Resultados: un grupo multiprofesional en salud con aproximadamente cinco años de actuación en la preceptoria, con experiencia en informática, aprobaron en casi $80 \%$ el uso del sistema en varios aspectos. Conclusión: el uso de recursos digitales dinámicos y interactivos, considerando la necesidad de un instrumento guía para las prácticas supervisadas en laboratorio clínico, la utilización de recursos digitales dinámicos e interactivos se muestra como alternativa para incrementar el proceso de aprendizaje.

Palabras-clave: Aplicación; Mobile Learning; Tecnologías de la Información.

\section{INTRODUÇÃO}

A adoção generalizada, na sociedade brasileira, de dispositivos móveis (como celulares, smartphones e tablets), como ferramentas de comunicação e entretenimento está redefinindo a forma como as pessoas interagem, se comportam e pensam. São comuns as consultas a aplicativos do tempo, do trânsito, de finanças, entre outros, para tomar pequenas decisões na vida cotidiana. O conforto de escolher onde usar e a facilidade de se deslocar no espaço físico, mantendo a conectividade, parecem representar novas formas de se informar e se comunicar. ${ }^{1}$

A disseminação desse tipo de dispositivo tem impulsionado o desenvolvimento de novos aplicativos (Apps) com potencial para incrementar o aprendizado dentro e fora da sala de aula. Há diversos tipos de Apps disponíveis, tais como, jogos, mídias sociais, livros (entre eles, dicionários, enciclopédias) e revistas, bem como aplicativos específicos para a educação, para a navegação na internet, para gerenciamento e organização de atividades e processos. ${ }^{2}$ 
A aprendizagem móvel viabiliza o espaço de convergência da Internet com as telecomunicações, criando ampla rede de comunicação e de oportunidades de aprendizagem. Essa perspectiva reposiciona a sala de aula e todos os espaços fora dela como lugares possíveis para ensinar e aprender. ${ }^{3}$

Diante desse contexto tecnológico, considera-se importante a utilização de ambientes multimídia por meio de aplicativo, oferecendo subsídio para o ensino e a verificação da aprendizagem, durante estágio em laboratório clínico, onde, no momento da atividade prática, o estudante poderá acessar conceitos teóricos e resolver problemas, além de disponibilizar, ao preceptor, uma nova ferramenta tecnológica para a supervisão de aprendizagem durante o estágio. O objetivo deste estudo é desenvolver e avaliar um aplicativo multimídia em plataforma móvel para a verificação de aprendizagem em laboratório clínico durante estágio supervisionado.

\section{MÉTODO}

\section{Criação do Protótipo}

O protótipo Labhupaa, desenvolvido neste projeto (Figura 1), é um aplicativo de aprendizagem móvel que tem por objetivo auxiliar o preceptor na verificação de aprendizagem dos estudantes durante estágio supervisionado e, além disso, os estudantes poderão consultar informações, participar de quiz e atualizar conceitos acerca do estágio em laboratório clínico. O processo de desenvolvimento foi definido em três etapas descritas abaixo.

1. Concepção e definição do problema: etapa essencial para o desenvolvimento do projeto. A definição surgiu a partir da necessidade de aprimoramento do processo de aprendizagem em estágio, pela carência de ferramenta para tornar o entendimento dos conteúdos atraentes e de fácil assimilação e articulação com a prática.

2. Definição e desenvolvimento: a construção do protótipo foi realizada no site Marvel app. Trata-se de um serviço on-line que tem como objetivo ajudar a montar protótipos de aplicações para smartphones. As telas criadas (Figura 1) referem-se a:

- Interface de Login: permite o controle por meio da identificação do usuário mediante a inserção de um usuário e senha; 
- Interface Central: após realizar o acesso, é apresentada, ao usuário, a área central do ambiente. Nesta interface, são listadas as opções para navegação;

- Interface de Aprendizagem: material didático com o conteúdo dividido em tópicos;

- Quiz e ranking: participando dos testes, o usuário avalia seus conhecimentos ao passo que o preceptor verifica o nível de aprendizagem e, ao final, é fornecido o ranking;

3. Avaliação: foi construído um questionário tipo likert com o objetivo de avaliar quanto à usabilidade do protótipo, ou seja, o usuário avalia em que grau o aplicativo o auxiliará. O questionário foi dividido em duas partes: parte I, que tem por objetivo traçar o perfil dos participantes e parte II, com perguntas específicas sobre o aplicativo.

4. Amostra e avaliação do protótipo com usuários

5. A amostra selecionada foi por conveniência e os voluntários foram convidados por meio do WhatsApp a participar da pesquisa, recebendo o link do protótipo e o link do questionário. Após a verificação do protótipo e antes de iniciar as respostas ao questionário, era necessário o aceite do Termo de Consentimento. Após o aceite, os participantes eram direcionados para as respostas ao questionário.

6. O modelo de protótipo desenvolvido deve ser de fácil interação e apresentar-se atraente entre o usuário e o sistema de forma que alcance alta usabilidade. Conforme a International Organization for Standardization (ISO-9241-11: 20184), para a determinação de quanto um produto pode ser usável dentro de um contexto, é necessário medir o desempenho (eficácia e eficiência) e a satisfação do usuário de forma que, para a avaliação do desempenho, foram utilizadas medidas objetivas e, para a satisfação do usuário, medidas subjetivas.

7. A avaliação constituiu da aplicação de dois questionários, sendo um questionário antes do teste, denominado pré-teste, para conhecer e determinar o perfil e a formação do usuário, e um questionário pós-teste para obter informações sobre a opinião do usuário em relação ao uso do sistema. 
Figura 1. Protótipo Labhupaa

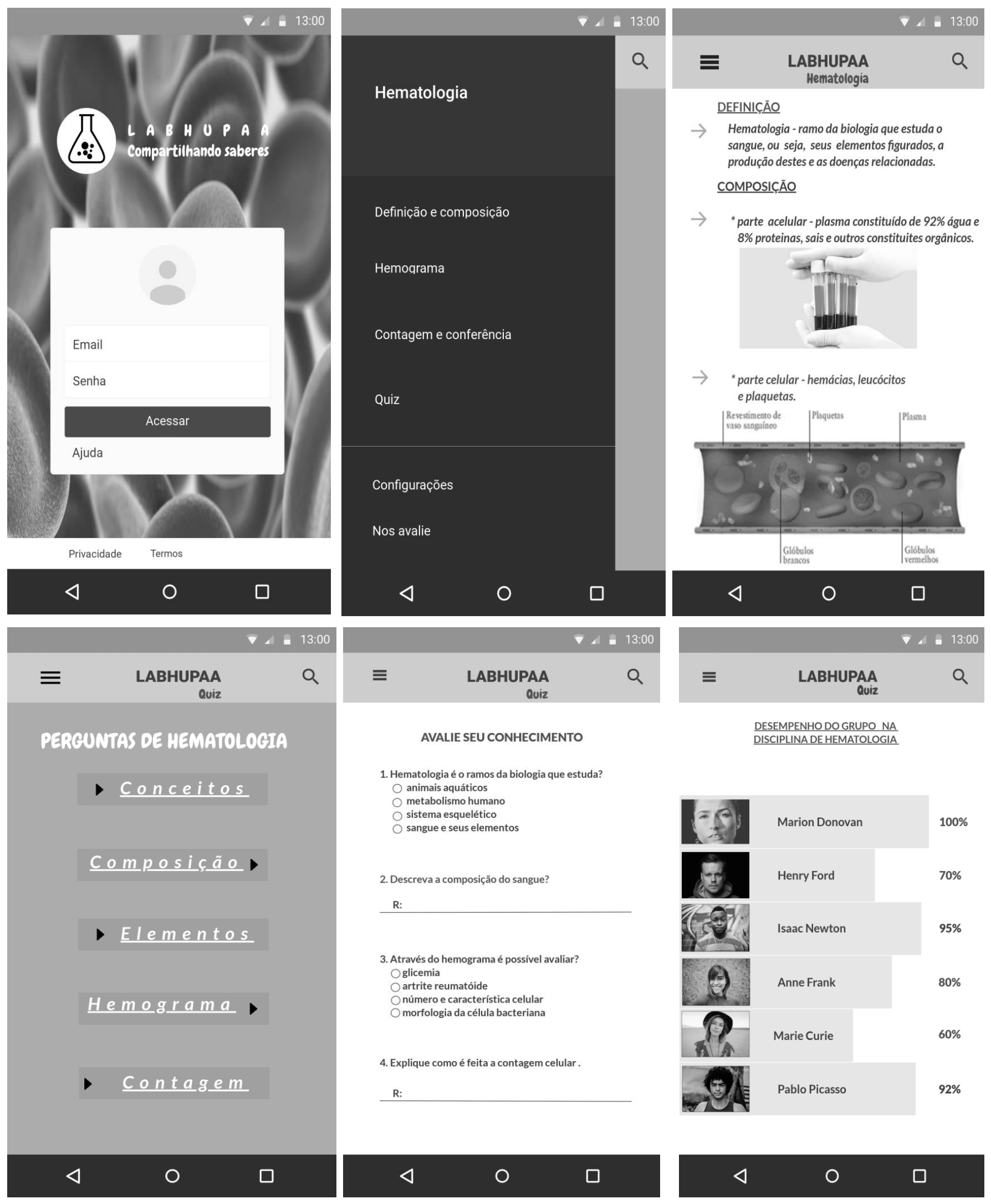

Fonte: Elaborado pelo autor via MarvelApp (2019). 
A parte I do questionário referiu-se ao perfil e à formação do participante. Composto de questões fechadas, propiciava a seleção de uma resposta dentre uma escolha entre alternativas. O questionário parte II, para obter informações sobre a opinião do usuário em relação ao uso do sistema, foi elaborado de forma a diminuir a carga de esforço e o tempo dispendido dos participantes, evitando que não completassem as respostas aos questionários.

A fim de não confundir o participante, o grau de concordância da escala foi adaptado para cinco itens, variando de um (discordo totalmente) a cinco (concordo totalmente). Duas questões abertas foram elaboradas a fim de permitir que o participante emitisse opinião sobre aspectos positivos e negativos do sistema.

\section{Análise Estatística}

Para a análise dos dados, inicialmente, foi criada uma planilha no programa Microsoft Excel for Windows 2007, com digitação e conferência dos mesmos. Os resultados das análises das variáveis foram expressos como média \pm desviopadrão e as variáveis categóricas, como frequência absoluta e porcentagem.

\section{RESULTADOS}

Vinte e um indivíduos foram convidados a participar, porém, vinte consentiram em participar da pesquisa. Dos 20 participantes com consentimento aceito, 19 responderam ao questionário. No questionário (parte I), as variáveis iniciais da amostra idade, sexo, formação (graduação), especialização e tempo de atuação na preceptoria de estágio estão representados no quadro 1.

\section{Quadro 1 - Questionário Parte I.}

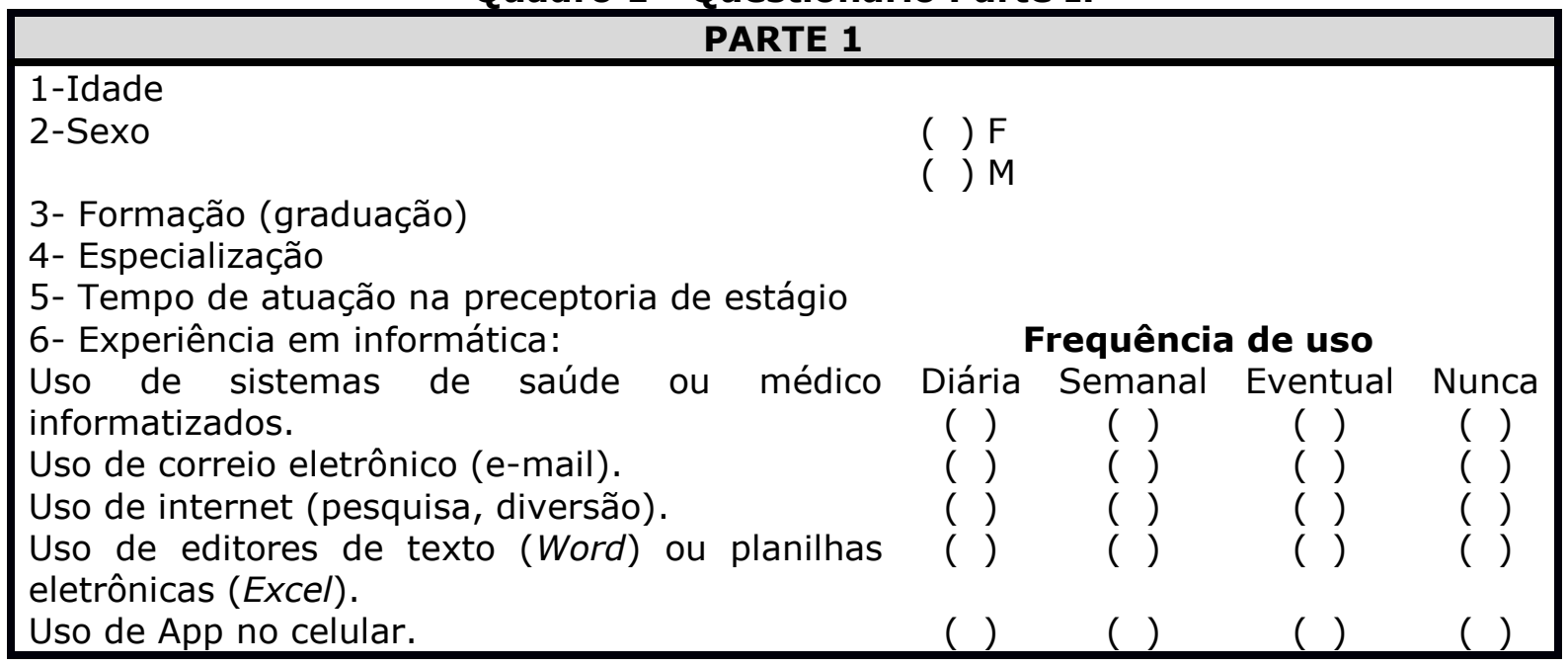


No item (Experiência em Informática) do questionário parte I, 100\% da amostra demonstrou experiência em informática com uso diário da internet para pesquisa/diversão. Quanto ao uso de sistemas de saúde ou médicos informatizados, 42,1\% usam diariamente.

Tabela 1. Opinião do Usuário em Relação ao Uso do Sistema.

\begin{tabular}{|c|c|c|c|c|c|c|}
\hline & & 5 & 4 & 3 & 2 & 1 \\
\hline \multicolumn{2}{|r|}{ A - REAÇÃO DO SISTEMA } & \multicolumn{5}{|c|}{$\%$} \\
\hline Q. 1 & Fácil entendimento & 94,7 & 5,3 & 0 & 0 & 0 \\
\hline Q. 2 & Fácil utilização & 84,2 & 10,6 & 5,2 & 0 & 0 \\
\hline Q. 3 & Funcionalidades são claras & 84,2 & 15,8 & 0 & 0 & 0 \\
\hline \multicolumn{7}{|c|}{ B - TELA } \\
\hline Q. 4 & $\begin{array}{l}\text { Forma e tamanho das letras estão de fácil } \\
\text { leitura }\end{array}$ & 89,5 & 5,3 & 5,2 & 0 & 0 \\
\hline Q. 5 & Organização da informação está clara & 89,5 & 10,5 & 0 & 0 & 0 \\
\hline Q. 6 & Sequência de telas está clara & 78,9 & 21,1 & 0 & 0 & 0 \\
\hline Q. 7 & Itens da tela são fáceis de encontrar & 89,5 & 10,5 & 0 & 0 & 0 \\
\hline \multicolumn{7}{|c|}{ C - APRENDIZADO } \\
\hline Q. 8 & Foi fácil aprender a operar o sistema & 84,2 & 15,8 & 0 & 0 & 0 \\
\hline Q. 9 & $\begin{array}{l}\text { O aplicativo poderá contribuir com o } \\
\text { aprendizado }\end{array}$ & 94,7 & 5,3 & 0 & 0 & 0 \\
\hline Q. 10 & $\begin{array}{l}\text { As atividades podem ser executadas de } \\
\text { maneira rápida e/ou lógica }\end{array}$ & 68,4 & 26,3 & 5,3 & 0 & 0 \\
\hline Q. 11 & $\begin{array}{l}\text { A conclusão do questionário final - Quiz - } \\
\text { estava clara }\end{array}$ & 57,9 & 21,1 & 15,8 & 5,2 & 0 \\
\hline \multicolumn{7}{|c|}{ D - CAPACIDADE DO SITEMA } \\
\hline Q. 12 & A velocidade do sistema é rápida & 63,2 & 21,1 & 10,5 & 0 & 5,2 \\
\hline Q. 13 & $\begin{array}{l}\text { Foi projetado facilmente para todos os níveis } \\
\text { de usuários (iniciantes e experientes) }\end{array}$ & 63,2 & 36,8 & 0 & 0 & 0 \\
\hline \multicolumn{7}{|c|}{ E - IMAGENS } \\
\hline Q. 14 & A qualidade das imagens está boa? & 89,5 & 5,3 & 5,2 & 0 & 0 \\
\hline Q. 15 & O tamanho das imagens está adequado? & 73,7 & 21,1 & 5,2 & 0 & 0 \\
\hline \multicolumn{7}{|c|}{ F - TERMINOLOGIA E INFORMAÇÃO DO SISTEMA } \\
\hline Q. 16 & $\begin{array}{l}\text { As mensagens que aparecem na tela são } \\
\text { claras }\end{array}$ & 63,2 & 36,8 & 0 & 0 & 0 \\
\hline Q. 17 & A localização das informações na tela é clara & 73,7 & 21,1 & 5,2 & 0 & 0 \\
\hline Q. 18 & As instruções aos usuários são claras & 65 & 15 & 15 & 5 & 0 \\
\hline \multicolumn{7}{|c|}{ G - UTILIDADE DO SITEMA } \\
\hline Q. 19 & $\begin{array}{l}\text { Esse sistema aprimoraria o meu trabalho } \\
\text { como preceptor }\end{array}$ & 72,2 & 22,2 & 5,6 & 0 & 0 \\
\hline Q. 20 & $\begin{array}{l}\text { Esse sistema tornaria o trabalho como } \\
\text { preceptor mais interessante }\end{array}$ & 79 & 10,5 & 10,5 & 0 & 0 \\
\hline Q. 21 & $\begin{array}{l}\text { Gostaria de ter esse sistema no meu dia a } \\
\text { dia como preceptor }\end{array}$ & 73,7 & 15,8 & 10,5 & 0 & 0 \\
\hline Q. 22 & $\begin{array}{l}\text { Eu usaria esse sistema se estivesse } \\
\text { disponível }\end{array}$ & 78,9 & 21,1 & 0 & 0 & 0 \\
\hline
\end{tabular}


No uso de e-mail, 89,5\% utilizam diariamente e 57,9\% utilizam editores de texto (Word) ou planilhas eletrônicas (Excel) diariamente.

Na parte II do questionário, quanto à opinião do participante em relação ao sistema, as respostas variaram de um (discordo totalmente) a cinco (concordo totalmente), sendo que a média geral das respostas dos que concordavam totalmente com as funções perguntadas foi de $77,8 \%$ e $0,23 \%$ discordavam totalmente, conforme demonstrado na tabela 1.

Nas perguntas abertas sobre os aspectos positivos do sistema, as palavras que se repetiram em maior número foram "fácil de utilizar" e "praticidade". Sobre os pontos negativos, a palavra que se repetiu mais vezes foi "velocidade do sistema (telas passam rápido demais)".

\section{DISCUSSÃO}

Até o fim dos anos 90, comprar um livro ou aprender um novo idioma dependia de um espaço físico para a criação de uma loja ou de uma escola. A evolução da tecnologia da informação, entretanto, possibilitou novas maneiras de venda de produtos e prestação de serviços. Assim, as facilidades que a evolução da tecnologia trouxe permitiram a criação de novos modelos de negócios como, também, novas maneiras de estudo e aprendizagem. ${ }^{5}$

O uso das Tecnologias de Informação e Comunicação (TIC) tem transformado significativamente os campos profissionais. Na área da educação, essa evolução tecnológica tem impulsionado o processo de aprendizagem. Hoje em dia, praticamente todos possuem um telefone celular. Com o avanço da internet, ampliaram-se as possibilidades de uso das tecnologias digitais como forma de obter informações das mais diversas. Os dispositivos são portáteis, têm preços acessíveis e oferecem diversos recursos que podem ser aproveitados também para a aprendizagem.

Os dispositivos móveis com conexão sem fio e interface sensível ao toque (touch-screen), tais como tablets e smartphones, associados a diferentes aplicativos, têm proporcionado mudanças na forma de as pessoas se relacionarem com a informação e produzir conhecimento, apresentando significativo potencial para transformar a maneira de ensinar e de aprender. ${ }^{2}$ Eles proporcionam, aos professores e estudantes, mobilidade e interface fáceis de usar, podendo, assim, contribuir para implementar diferentes estratégias de ensino e de aprendizagem. 
Dessa forma, para os estudantes de hoje, os chamados nativos digitais, torna-se mais atraente estudar fazendo uso de tais dispositivos, uma vez que a moderna tecnologia os acompanha desde o nascimento. ${ }^{5}$ Assim, o mobile learning ou aprendizagem móvel surge como possibilidade educativa, favorecendo o acesso à informação e à aprendizagem por meio de atividades conectadas, como treinamentos, cursos, aulas virtuais, entre outras, propiciando a comunicação multidirecional com os participantes.

O ambiente de aprendizagem deixa de ser só a sala de aula, expandindo-se para todos os lugares, em uma sincronia do ensino híbrido educacional, presencial e virtual/digital, voltando-se a uma era digital associada à informática, computadores, cibernética e da conectividade. ${ }^{6}$

A diversidade de cenários de práticas (estágios) tem apresentado crescente importância nos últimos anos para a formação de recursos humanos em saúde, tornando-se essencial para o ensino e a aprendizagem, e as instituições de ensino estão cada vez mais fazendo uso da tecnologia para apoio ao ensino, propiciando troca de saberes entre estudantes, professores e preceptores. Portanto, entendese que o mobile learning possibilita maior controle e autonomia do estudante sobre sua aprendizagem, em um contexto de contínua conectividade, de forma espontânea e conveniente, favorecendo o processo de ensino-aprendizagem, avaliação e feedback de forma inovadora.

Conforme exposto, este artigo apresenta uma propositura de desenvolvimento de um protótipo de aplicativo mobile voltado para a avaliação de aprendizagem em laboratório clínico durante estágio supervisionado. Esse protótipo visa a facilitar e auxiliar o aprendizado dos estudantes, possibilitando o acompanhamento do aprendizado pelo preceptor, que poderá avaliar, de forma simples e rápida, o nível de aprendizagem dos estudantes, proporcionando uma melhor interação e troca de conhecimentos para melhor aproveitamento do estágio.

\section{CONCLUSÃO}

Mostra-se a utilização de recursos digitais dinâmicos e interativos, considerando a necessidade de um instrumento norteador para os estágios supervisionados em laboratório clínico, como alternativa para aprimorar o processo ensino-aprendizagem. A teoria construtivista da aprendizagem tem sido a abordagem mais utilizada para nortear o desenvolvimento de recursos 
educativos informatizados. Neste sentido, surgiu a proposta para a construção do aplicativo, podendo oferecer ao estudante a oportunidade de melhorar a relação teoria/prática, estabelecendo uma correlação entre o que aprende e as situações reais, experimentais, tornando a aprendizagem enriquecedora. Em outra perspectiva, é uma ferramenta pedagógica que subsidia o preceptor no

\section{REFERÊNCIAS}

1. Pina F, Kurtz R, Ferreira JB, Freitas A, Silva JF, Giovannini CJ. M-Learning adoption in higher education: the professor's point of view. Read Rev Eletrônica Adm. 2016 May/Aug; 22(2):279-306. Doi: http://dx.doi.org/10.1590/14132311.0262015 .54352$.

2. Nichele AG, Schlemmer E. Aplicativos para o ensino e aprendizagem de Química. Rev Renote- Novas Tecnologias na Educação. 2014; 2:01-09. Doi: https://doi.org/10.22456/1679-1916.53497

3. Melo RS, Carvalho MJS. Aplicativos educacionais livres para mobile learning. XI Evidosol e VIII Ciltec [Internet]. 2014 June [cited 2018 Aug 10]; 2: 1-6. Available from:

http://www.periodicos.letras.ufmg.br/index.php/anais_linguagem_tecnologia/artic le/view/5809/5098

4. Associação Brasileira de Normas Técnicas. NBR ISO 9241-11. Define usabilidade e explica como identificar a informação necessária a ser considerada na especificação ou avaliação de usabilidade de um dispositivo de interação visual em termos de medidas de desempenho e satisfação do usuário. Orientação é dada sobre como descrever o contexto de uso do produto (hardware, software ou serviços) e as medidas relevantes de usabilidade de uma maneira explícita. A orientação é dada na forma de princípios e técnicas gerais, em vez da forma de requisitos para usar métodos específicos. Rio de Janeiro: ABNT; 2011.

5. Martins WS, Allevato NSG, Dias KM, Schimiguel J, Pires CMC. M- learning as a method of education: the use of the Estatística Fácil application in High School. Ensino da Matemática em Debate [Internet]. 2018 [cited 2018 Aug 10]; 5(1):0117. Available from: https://revistas.pucsp.br/index.php/emd/article/view/32882

6. Santos TS. Tecnologia e educação: o uso de dispositivos móveis em sala de aula [dissertation] [Internet]. Londrina: Universidade Tecnológica Federal do Paraná; 2016 [cited 2018 Aug 10]. Available from: http://repositorio.roca.utfpr.edu.br/jspui/bitstream/1/7491/1/LD_ENT_II_2016_1 8.pdf 